\section{Caracterização dos usuários e dos serviços prestados por Centros de Atenção Psicossocial Infanto-Juvenil}

\author{
Characteristics of individuals and care delivered \\ at the Psychosocial Care Centers for Children and \\ Adolescents
}

\author{
${ }^{1}$ Ministério da Saúde, \\ Brasília, Brasil. \\ 2 Instituto de Saúde Coletiva, \\ Universidade Federal da \\ Bahia, Salvador, Brasil. \\ Correspondência \\ M. C. C. L. Hoffmann \\ Coordenação-Geral de Gestão \\ do Conhecimento, \\ Ministério da Saúde. \\ Esplanada dos Ministérios, \\ Bloco G, 8o andar, sala 851, \\ Brasília, DF \\ 70.900-500, Brasil. \\ cristina.hoffmann@ig.com.br
}

\section{Abstract}

This study aimed to provide an understanding of diagnosis, demographic characteristics, and treatment procedures for children and adolescents attending Psychosocial Care Centers (CAPSIS) in Brazil, 2003. It was a cross-sectional descriptive study using secondary data. The analysis considered three groups of variables related to: (a) the nature of the services; (b) patient profile; and (c) types of care. Seven CAPSIS that had recorded their procedures during all 12 months of 2003 participated in the study. The units involved treated 1,456 individuals, 62.8\% of whom were males 11-13 years of age. Nearly half $(49.3 \%)$ were treated in non-intensive care. The most frequent diagnoses were behavioral and emotional disorders (44.5\%). There was no record of treatment related to use of psychoactive substances. The proportion of severe disorders was 19.4\%. The CAPSIS operate in similar fashion to older outpatient units, but treat a variety of problems, creating a record according to types of activities. These services fill a historical gap in public mental health care for children and adolescents.

Mental Health Services; Child; Adolescent; Mental Health
Maria Cristina Corrêa Lopes Hoffmann 1

Darci Neves Santos 2

Eduardo Luiz Andrade Mota 2

\section{Introdução}

O reduzido número de publicações em nosso meio sobre problemas de saúde mental na infância e adolescência e sobre a utilização de serviços por esta população tem sido demonstrado 1 , dificultando o planejamento das ações de saúde pública neste setor.

Estudos internacionais têm registrado prevalências entre $10 \%$ e $20 \%$, de um ou mais problemas mentais na população infanto-juvenil, sendo mais freqüentes os transtornos do desenvolvimento psicológico, transtornos emocionais e de comportamento 2,3 .

A primeira informação epidemiológica em saúde mental infantil no Brasil surgiu em 1982, indicando uma prevalência de $23,5 \%$ para algum tipo de transtorno em crianças de 5 a 14 anos 4 residentes em Salvador, Bahia. Um segundo estudo de base populacional com escolares entre 7 e 14 anos constatou uma prevalência de $12,7 \%$ de transtornos mentais no Município de Taubaté, São Paulo ${ }^{5}$. A taxa de prevalência para um ou mais transtornos psiquiátricos nesta mesma faixa etária variou de $7 \%$ a $12,7 \%$, conforme estudo realizado em Ilha de Maré, Bahia 6 .

Estudo multicêntrico conduzido em São Paulo, Brasília e Porto Alegre (Rio Grande do Sul) estimou prevalências de sintomatologia depressiva no grupo etário de 15 a 19 anos, encontrando valores de $8 \%$ a $12 \%$ para inapetência, $6 \%$ a $8 \%$ para insônia, $11 \%$ a $17 \%$ para desânimo e $7 \%$ a $15 \%$ 
para irritabilidade; ideação suicida variou entre $3 \%$ e $4 \% 7$. A literatura assinala maior susceptibilidade para o quadro depressivo entre adolescentes mais velhos e do sexo feminino ${ }^{8}$. Entre crianças e adolescentes usuários de um Centro de Atenção Psicossocial do Rio Grande do Sul houve um predomínio de pessoas do sexo masculino, porém os diagnósticos de transtornos de humor e transtornos neuróticos relacionados ao estresse foram mais freqüentes entre as mulheres 9 .

Apesar da magnitude dos problemas, a atenção pública em saúde mental dirigida ao grupo infanto-juvenil tem sido marcada por um vazio histórico em sua cobertura, preenchido ao longo dos anos por instituições, na sua maioria de natureza privada ou filantrópica, tornando-se, em algumas localidades, as únicas opções de cuidado disponível às crianças, jovens e seus familiares. Ademais, a partir da década de 1920, a tendência à psiquiatrização e medicalização de problemas de ordem comportamental ou educacional adotou como diretriz a suposição de que muitas famílias não apresentavam condições de cuidar e de se responsabilizar pelo bem estar de seus filhos, levando muitas crianças e jovens à internação em abrigos e educandários 10,11.

Embora alternativas àquele modelo de atenção tivessem surgido na década de $80 \mathrm{com}$ os primeiros Núcleos e Centros de Atenção Psicossocial (NAPS e CAPS) 12, apenas a partir de 2002 foram implantados serviços públicos específicos para crianças, adolescentes e jovens, denominados Centros de Atenção Psicossocial Infanto-Juvenis (CAPSI) 13. Tais serviços foram propostos com a função de prover a atenção comunitária em saúde mental de forma integral, além de organizar a rede de cuidado existente no seu território de abrangência, tornando-se, portanto, elementos estratégicos nesta proposta de reversão do modelo hospitalocêntrico. Os CAPSI foram planejados para atender prioritariamente às demandas de transtornos psiquiátricos severos e persistentes, empregando alternativas de tratamento apoiadas em uma proposta terapêutica individualizada, articulada com diferentes serviços extra-hospitalares como residências terapêuticas, ambulatórios, leitos em hospitais gerais, oficinas de geração de renda, entre outros. O papel de ordenador da rede exige que os CAPSI sejam de natureza jurídica pública, identificando o hospital geral ou pediátrico do território onde se insere como local de acolhimento perante a necessidade de internação 14,15.

Cerca de 3\% da população geral, independente de faixa etária, necessita de cuidados contínuos em saúde mental por apresentarem transtornos mais severos e persistentes como psicoses, neuroses graves, transtornos de humor e autis- mo 16. Considerando esse percentual, estima-se que mais de 2 milhões dentre os 74 milhões de brasileiros com menos de 21 anos pudessem se beneficiar com a implantação de tal rede de atenção pública em saúde mental. Procura-se com este estudo identificar o perfil epidemiológico da clientela atendida nos CAPSI recentemente implantados e descrever algumas características dos serviços, com o intuito de verificar os atendimentos registrados no primeiro ano de funcionamento e se esses CAPSI atendem os problemas mentais severos para os quais foram planejados.

\section{Métodos}

Trata-se de um estudo descritivo, de corte transversal, realizado com dados secundários obtidos dos registros de atendimento realizados no ano de 2003 pelos CAPSI habilitados no ano anterior, junto ao Sistema Único de Saúde (SUS). Em 2002, foram habilitados 32 CAPSI; contudo, somente sete foram incluídos no estudo porque registraram os atendimentos durante todos os meses do ano estudado no Sistema de Informações Ambulatoriais (SIA).

Quatro CAPSI, um de Brasília outro do Piauí e dois do Rio de Janeiro não apresentaram nenhum registro durante todo o ano estudado, enquanto 21 unidades não completaram os 12 meses de registro, estando 5 delas no Município de São Paulo. As 16 unidades restantes localizaram-se individualmente nas cidades de Goiânia (Goiás), Betim (Mato Grosso), Belém (Pará), Cascavel (Paraná), Recife (Pernambuco), Volta Redonda (Rio de Janeiro), Barra Mansa (Rio de Janeiro), Rio de Janeiro, Porto Alegre (Rio Grande do Sul), Blumenau (Santa Catarina), Itajaí (Santa Catarina), Campinas (São Paulo), São José dos Campos (São Paulo) e Bauru (São Paulo). As sete unidades participantes do estudo geraram 8.539 registros referentes ao atendimento de 1.456 pessoas enquanto as 21 unidades com dados insuficientes para os 12 meses geraram 9.749 registros referentes ao atendimento de 2.292 pessoas, $61,16 \%$ do total de pessoas (3.748) atendidas nos 28 CAPSI com algum registro em 2003. Nos CAPSI excluídos, a média de idade foi 12,3 anos (desvio padrão - DP = 3,86 ), com $67 \%$ das pessoas do sexo masculino, figurando neste grupo o único CAPSI que registrou problemas decorrentes do uso de álcool e outras drogas.

Os dados utilizados para a caracterização demográfica e clínica da clientela foram gerados pelas Autorizações de Pagamento de Serviços de Alta Complexidade (APAC), sob gestão do Departamento de Informática do SUS (DATASUS), Ministério da Saúde. Os bancos de dados foram 
disponibilizados pelo Departamento de Regulação, Avaliação e Controle (DRAC) da Secretaria de Atenção à Saúde do Ministério da Saúde, mediante solicitação por escrito dos pesquisadores. As características administrativas dos CAPSI foram descritas com base nos dados do Cadastro Nacional de Estabelecimentos de Saúde (CNES), por consulta à página eletrônica http://www. cnes.datasus.gov.br, realizada em 24 de julho de 2005.

A análise foi realizada de acordo com três conjuntos de variáveis, a saber:

a) variáveis relativas à natureza dos serviços: 1 . localização (município e estado); 2. esfera administrativa (pública municipal, pública estadual ou filantrópica); 3. tipo de gestão (municipal, estadual) e 4. equipe completa (um médico, um enfermeiro, quatro profissionais de nível superior e cinco profissionais de nível médio), segundo as diretrizes da Portaria $n^{\circ}$. 336/2002 13;

b) variáveis relativas à clientela: idade, sexo, município de residência e diagnóstico inicial recebido, de acordo com a Classificação Estatística Internacional de Doenças e Problemas Relacionados à Saúde, 10a Revisão (CID-10) 17. Baseado em publicação do Fundo de População das Nações Unidas (UNFPA) 18 que considera infância as faixas etárias até 10 anos incompletos, adolescência, entre 10 e 19 anos e juventude o período entre 15 e 24 anos, optou-se por agrupar a clientela em três grupos etários: infância (até 10 anos), adolescência (11 a 14 anos) e juventude (15 a 21 anos). Foi tomado como limite máximo para a análise a idade de 21 anos, considerando o limite etário para o atendimento nos CAPSI, segundo as normas do Ministério da Saúde 13;

c) variável relativa ao cuidado oferecido: refere-se à modalidade de atendimento em que o paciente foi engajado, sendo definida em três categorias: intensivo, quando a freqüência ao serviço ocorria cinco dias por semana; semi-intensivo, se apenas três dias por semana; não intensivo, se quinzenal ou três vezes por mês.

Todas as informações foram processadas no pacote estatístico Epi Info versão 3.3.2 (Centers for Disease Control and Prevention, Atlanta, Estados Unidos), realizando-se análises univariada, bivariada e estratificada. Calculou-se a razão de freqüências relativas para as estimativas de efeito e aplicou-se o teste qui-quadrado para testar a significância estatística das diferenças entre proporções, adotando-se valor de alfa igual ou menor que 0,05.

Para verificar associação entre diagnóstico e grupos etários, tomou-se como grupo de referência os menores de 10 anos, e entre diagnóstico e sexo, os indivíduos do sexo feminino.
O tempo médio de permanência em atendimento no ano do estudo foi estabelecido pela média entre o primeiro e o último atendimento registrado em 2003, calculando-se o coeficiente de variação (DP/média x 100) para verificar a representatividade das médias.

Embora tenha sido um estudo realizado com dados secundários, foi obtido acesso aos dados individualizados. Por esta razão, foi esclarecido no termo de responsabilidade submetido à instituição-fonte dos dados a declaração de preservação do anonimato dos usuários e a apresentação das informações somente de forma consolidada. O estudo foi aprovado pelo Comitê de Ética do Instituto de Saúde Coletiva da Universidade Federal da Bahia em 5 de maio de 2005.

\section{Resultados}

\section{Caracterização dos CAPSI do estudo}

Os sete serviços estudados estavam distribuídos por três regiões, sendo quatro no Sudeste: um em São Paulo (Sorocaba) e três em Minas Gerais (Contagem, Uberaba e Uberlândia); dois na Região Sul: no Estado do Paraná (Cambé e Londrina); e um na Região Centro-Oeste, em Mato Grosso (Cuiabá).

Existiam cinco serviços municipais, um estadual e um filantrópico. Já em relação à gestão, cinco encontravam-se sob gestão municipal e dois sob gestão estadual. Os dados do CNES indicaram que apenas o CAPSI de Contagem apresentou equipe conforme o preconizado nas normas vigentes. As diferenças em relação às normas referiam-se ao número menor de profissionais de nível médio e do profissional de enfermagem, cujo registro estava ausente em um dos serviços estudados.

\section{Características da clientela}

Do total de 1.456 pessoas atendidas nos sete CAPSI, $62,8 \%$ eram do sexo masculino e a média de idade foi de 11,1 anos, (DP = 3,9; variação de $<1$ a 54) (Tabela 1). Verificou-se o registro de atendimento a cinco pessoas maiores de 21 anos $(0,3 \%)$, e quatro foram atendidas em um único serviço, localizado em Contagem e uma em Cuiabá.

\section{Características do atendimento}

A mediana do número de atendimentos anuais foi de 238 pessoas (intervalo de 59 a 370 pessoas). Do total de usuários, $49,3 \%$ estavam vinculados à modalidade não intensiva, $40,2 \%$ à semi-inten- 
Distribuição de idade e número de pessoas atendidas em sete Centros de Atenção Psicossocial Infanto-Juvenis (CAPSI) selecionados conforme município e Unidades da Federação (UF). Brasil, 2003.

\begin{tabular}{|c|c|c|c|c|c|c|c|}
\hline \multirow[t]{2}{*}{ UF } & \multirow[t]{2}{*}{ Município } & \multicolumn{2}{|c|}{ Pessoas atendidas } & \multicolumn{4}{|c|}{ Idade (anos) } \\
\hline & & $\mathrm{n}$ & $\%$ & Média & Desvio padrão & Mínima & Máxima \\
\hline Minas Gerais & Contagem & 232 & 16,0 & 12,9 & 4,2 & 3 & 23 \\
\hline Minas Gerais & Uberaba & 153 & 10,5 & 10,8 & 3,5 & 2 & 18 \\
\hline Minas Gerais & Uberlândia & 59 & 4,0 & 9,7 & 3,6 & 4 & 20 \\
\hline Mato Grosso & Cuiabá & 133 & 9,1 & 9,8 & 4,9 & 3 & 54 \\
\hline Paraná & Cambé & 370 & 25,4 & 11,2 & 3,6 & $<1$ & 19 \\
\hline Paraná & Londrina & 265 & 18,2 & 12,4 & 3,2 & 5 & 18 \\
\hline São Paulo & Sorocaba & 243 & 16,7 & 9,1 & 2,6 & 1 & 14 \\
\hline Total & & 1.455 & 100,0 & 11,1 & 3,9 & $<1$ & 54 \\
\hline
\end{tabular}

Fonte: Banco de dados Sistema de Informações Ambulatoriais/Autorizações de Pagamento de Serviços de Alta Complexidade, Departamento de Informática do SUS, Ministério da Saúde, 2003.

Nota: O CAPSI de Cuiabá teve um caso de idade não identificada.

siva e 10,5\% à intensiva (Tabela 2). O CAPSI de Uberaba não registrou nenhuma pessoa no procedimento não intensivo, no entanto, $97,4 \%$ das pessoas atendidas foram vinculadas ao procedimento semi-intensivo.

Observou-se diferença entre os serviços quanto ao número mensal de atendimentos por tipo de procedimento. No procedimento intensivo, o CAPSI de Sorocaba apresentou a maior média de pessoas atendidas por mês, 25,5 registros, e o CAPSI de Uberaba a menor média, de 1,7 registro por mês.

Em relação aos atendimentos realizados no procedimento semi-intensivo, o CAPSI de Uberaba apresentou a maior média com 78,6 registros mensais; já o CAPSI de Cuiabá apresentou a menor média, com 21,3 registros. No procedimento não intensivo Sorocaba, novamente, apresentou a maior média, com 84,6 registros mensais, e Uberlândia a menor média, com 5,3 registros. O percentual da clientela residente em cada município sede da unidade de atendimento atingiu $100 \%$ em Cambé, Contagem e Uberaba, aproximadamente 99\% em Londrina e Uberlândia, 96,7\% em Sorocaba, chegando a 88,15 em Cuiabá.

\section{Perfil diagnóstico}

Em torno de $78 \%$ dos diagnósticos concentraram-se em três grupos da CID-10 17. Encontrou-se um percentual de $44,5 \%$ para o grupo de transtornos do comportamento e transtornos emocionais (F90-F98), seguido por 19,8\% para transtornos neuróticos (F40-F49) e 14,2\% para transtornos do desenvolvimento psicológico
(F80-F89) (Tabela 3). Ao associar o diagnóstico com a idade, verificou-se que os transtornos do comportamento e transtornos emocionais foram os mais freqüentes nos três grupos etários, apresentando as freqüências de $46,9 \%$ entre menores de 10 anos; 47,6\% para o grupo de 11 a 14 anos; e $32,7 \%$ para aqueles entre 15 e 21 anos.

Observou-se, ainda, que quase não houve diferença quando comparados os grupos menores de 10 e o de 11 a 14 anos, exceto em relação ao diagnóstico dos transtornos do desenvolvimento psicológico, em que os menores de 10 anos apresentaram maior freqüência, sendo esta estatisticamente significante. Já em relação às comparações realizadas entre os menores de 10 e o grupo de 15 a 21, as diferenças foram significantes para a maioria dos diagnósticos, com exceção dos transtornos neuróticos e síndromes comportamentais.

Os diagnósticos de esquizofrenia, transtornos de humor e retardo mental tiveram maior freqüência entre os mais velhos e tal diferença foi estatisticamente significante, destacando-se a razão entre freqüências relativas para o diagnóstico de esquizofrenia (razão entre freqüências relativas $-\mathrm{RF}=15-21 /<10=7$ ). Os transtornos do desenvolvimento psicológico, do comportamento e os não especificados foram mais freqüentes entre os menores de 10 do que entre aqueles do grupo de 15 a 21 anos $(\mathrm{RF}=15-21 /<10=0,29)$ (Tabela 3).

Os transtornos graves como psicoses, transtornos de humor e transtornos globais do desenvolvimento representaram $19,4 \%$ do total de diagnósticos (dados não mostrados em tabela). 
Distribuição percentual (\%) dos atendimentos por modalidade, segundo municípios. Brasil, 2003.

\begin{tabular}{|c|c|c|c|c|c|c|c|c|}
\hline Modalidades de atendimento & Cambé & Londrina & Cuiabá & Contagem & Uberaba & Uberlândia & Sorocaba & Total \\
\hline Intensivo & 17,6 & 2,6 & 16,4 & 6,0 & 2,6 & 8,50 & 14,8 & 10,5 \\
\hline Semi-itensivo & 27,6 & 36,2 & 32,1 & 34,1 & 97,4 & 74,6 & 29,6 & 40,2 \\
\hline Não intensivo & 54,9 & 61,1 & 51,5 & 59,9 & 0,0 & 16,9 & 55,6 & 49,3 \\
\hline
\end{tabular}

Fonte: Banco de dados Sistema de Informações Ambulatoriais/Autorizações de Pagamento de Serviços de Alta Complexidade, Departamento de Informática do SUS, Ministério da Saúde, 2003.

Tabela 3

Número de casos e freqüências relativas dos diagnósticos recebidos pela clientela atendida nos Centros de Atenção Psicossocial Infanto-Juvenis (CAPSI), selecionados por ocasião do início do atendimento, segundo três grupos etários. Brasil, 2003.

\begin{tabular}{|c|c|c|c|c|c|c|c|}
\hline \multicolumn{2}{|r|}{ Diagnóstico } & \multirow{2}{*}{$\begin{array}{l}\text { Total } \\
\text { n (\%) }\end{array}$} & \multirow{2}{*}{$\begin{array}{c}<10 \text { anos } \\
n(\%)\end{array}$} & \multirow{2}{*}{$\begin{array}{c}11 \text { a } 14 \text { anos } \\
\text { n (\%) }\end{array}$} & \multirow{2}{*}{$\begin{array}{c}15 \text { a } 21 \text { anos } \\
n(\%)\end{array}$} & \multirow[t]{2}{*}{ Valor de $p$ * } & \multirow[t]{2}{*}{ Valor de $p^{* *}$} \\
\hline Código da CID-10 & Descrição & & & & & & \\
\hline F20-F29 & $\begin{array}{l}\text { Esquizofrenia, transtornos } \\
\text { esquizotípicos e delirantes }\end{array}$ & $43(3,0)$ & $9(1,4)$ & $8(1,5)$ & $26(9,8)$ & 0,858 & $<0,001$ \\
\hline F29-F30 & Transtornos de humor (afetivos) & $127(8,7)$ & $37(5,6)$ & $44(8,2)$ & $46(17,3)$ & 0,078 & $<0,001$ \\
\hline F40-F48 & $\begin{array}{l}\text { anstornos neuróticos, transtornos relacionados } \\
\text { com o estresse e transtornos somatoformes }\end{array}$ & $288(19,8)$ & $116(17,7)$ & $112(21,0)$ & $60(22,6)$ & 0,155 & 0,090 \\
\hline F50-F59 & $\begin{array}{l}\text { Síndromes comportamentais associadas } \\
\text { com distúrbios fisiológicos e a fatores físicos }\end{array}$ & $19(1,3)$ & $7(1,1)$ & $6(1,1)$ & $6(2,3)$ & 0,928 & 0,282 \\
\hline F70-F79 & Retardo mental & $83(5,7)$ & $30(4,6)$ & $30(5,6)$ & $23(8,6)$ & 0,416 & 0,016 \\
\hline F80-F89 & Transtornos do desenvolvimento psicológico & $207(14,2)$ & $126(19,2)$ & $66(12,4)$ & $15(5,6)$ & 0,001 & $<0,001$ \\
\hline F90-F98 & $\begin{array}{l}\text { Transtornos do comportamento e transtornos } \\
\text { emocionais que aparecem habitualmente } \\
\text { na infância ou na adolescência }\end{array}$ & $648(44,5)$ & $307(46,9)$ & $254(47,6)$ & $87(32,7)$ & 0,811 & $<0,001$ \\
\hline F99 & Transtorno mental não especificado & $40(2,7)$ & $23(3,5)$ & $14(2,6)$ & $3(1,1)$ & 0,380 & 0,048 \\
\hline Total & & $1.445(100,0)$ & $655(100,0)$ & $534(100,0)$ & $266(100,0)$ & & \\
\hline
\end{tabular}

CID-10: Classificação Estatística Internacional de Doenças e Problemas Relacionados à Saúde, 10ạ Revisão (CID-10) 17.

Fonte: Banco de dados Sistema de Informações Ambulatoriais/Autorizações de Pagamento de Serviços de Alta Complexidade, Departamento de Informática do SUS, Ministério da Saúde, 2003.

* Comparação entre grupo de 11 a 14 anos e grupo de < 10 anos:

** Comparação entre grupo de 15 a 21 anos e grupo de < 10 anos.

Nenhum dos CAPSI estudados registrou atendimento de pessoas com transtornos mentais e comportamentais devido ao uso de substâncias psicoativas.

Tomando-se sexo feminino como referência na comparação por diagnóstico e sexo, constataram-se associações significantes para a maioria dos diagnósticos, com exceção dos grupos de retardo mental e de transtornos mentais não especificados. Indivíduos do sexo feminino tiveram mais diagnósticos de transtornos de humor e transtornos neuróticos, em relação ao sexo masculino, sendo mais freqüente entre o último os diagnósticos de esquizofrenia, síndromes comportamentais, transtornos do desenvolvimento psicológico e transtornos do comportamento, diferenças estatisticamente significantes (Tabela 4). 
Número de casos, freqüências relativas e razão entre as freqüências relativas dos diagnósticos recebidos pela clientela atendida nos Centros de Atenção Psicossocial Infanto-Juvenis (CAPSI), selecionados por ocasião do primeiro atendimento, segundo o sexo. Brasil, 2003.

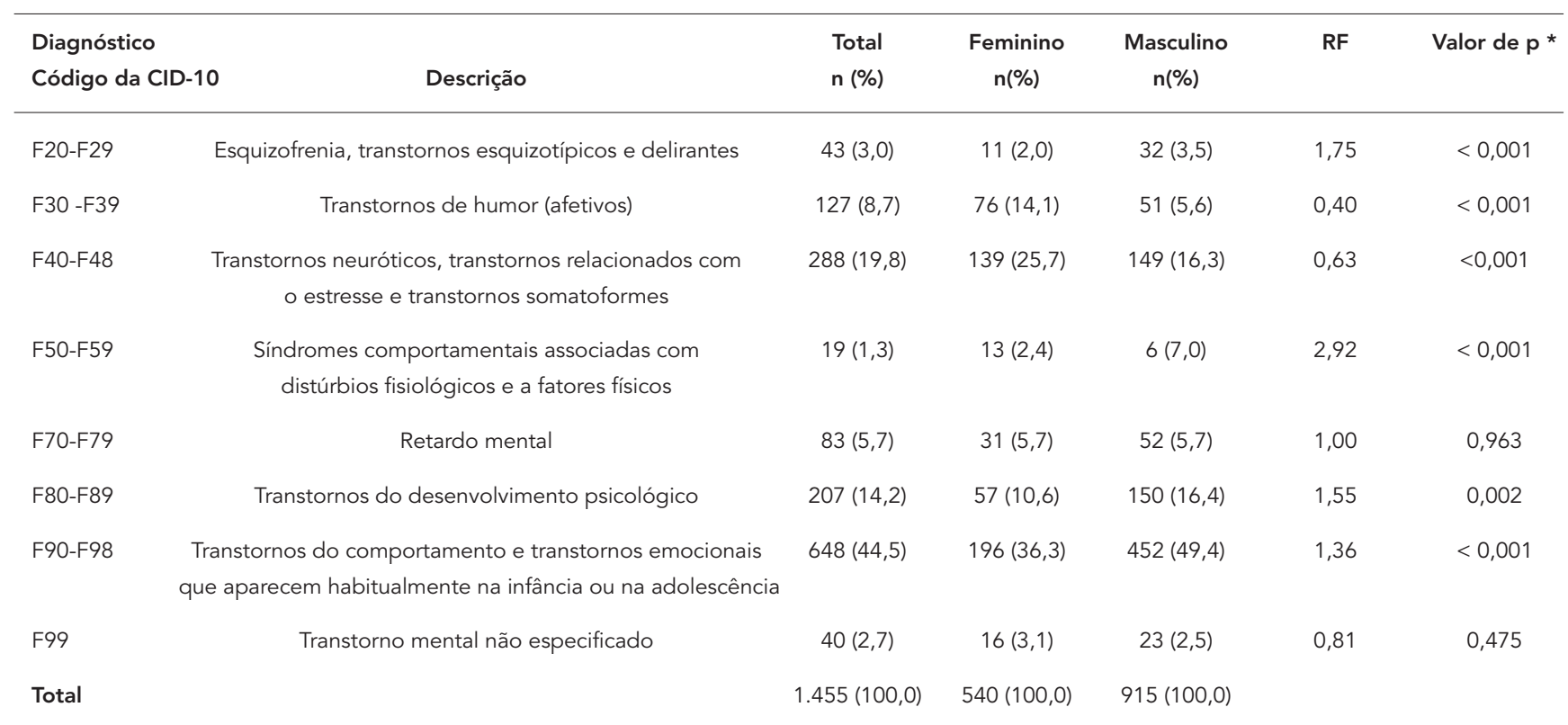

CID-10: Classificação Estatística Internacional de Doenças e Problemas Relacionados à Saúde, 10a Revisão (CID-10) 17; RF: razão entre freqüências relativas. Fonte: Banco de dados Sistema de Informações Ambulatoriais/Autorizações de Pagamento de Serviços de Alta Complexidade, Departamento de Informática do SUS, Ministério da Saúde, 2003.

* Comparação entre grupo masculino e feminino.

O tempo médio transcorrido entre a primeira e a última consulta registrada no ano de 2003, foi de 5,39 meses, ( $D P=3,8$ ). O grupo com diagnóstico de transtornos do desenvolvimento psicológico apresentou maior tempo médio entre a primeira e a última consulta, com coeficiente de variação de $54,83 \%$, enquanto o menor tempo médio foi observado no grupo com diagnóstico de transtorno do humor, cujo coeficiente de variação foi de $87,37 \%$. A mediana foi igual ou maior que sete meses para esquizofrenia, síndromes comportamentais associadas com distúrbios fisiológicos e transtornos do desenvolvimento psicológico (Tabela 5).

\section{Discussão}

Os resultados encontrados sugerem que os CAPSI estão atendendo uma clientela compatível com o que preconizam as normas relativas à faixa etária e o território de abrangência.

Os diagnósticos mais freqüentes concordaram com os achados da literatura 3 , que apontou os transtornos de comportamento como mais freqüentes, independente de grupo etário. A oferta desses serviços atende às necessidades de saúde assinaladas por estudos de prevalência sobre os principais problemas de saúde mental entre jovens brasileiros.

Concordando com a literatura os diagnósticos de esquizofrenia e de transtornos de humor, foram mais freqüentes no grupo etário de 15 a 21 anos do que em relação aos demais grupos, enquanto os transtornos do desenvolvimento psicológico, e do comportamento apresentaram maior freqüência entre os menores de 10 anos. Os transtornos neuróticos e de humor, foram mais freqüentes entre as jovens do sexo feminino, enquanto os transtornos de comportamento e do desenvolvimento psicológico foram mais freqüentes para o sexo masculino, achados também observados por outros estudos 7,9.

A elevada freqüência de diagnósticos relativos a problemas de habilidades escolares coloca a necessidade de investimento na articulação e discussão entre profissionais da saúde e da área educacional, com a finalidade de reduzir encaminhamentos para serviços especializados de atenção à saúde mental, como os CAPSI, perante dificuldades relacionadas exclusivamente aos problemas do aprendizado.

A baixa freqüência de diagnósticos por retardo mental $(5,7 \%)$ pode estar relacionada ao pro- 
Média em meses do tempo decorrido entre o primeiro e o último registro de atendimento nos Centros de Atenção Psicossocial Infanto-Juvenis (CAPSI), selecionados segundo o diagnóstico. Brasil, 2003.

\begin{tabular}{|c|c|c|c|c|}
\hline Diagnóstico & & Média & Desvio padrão & Mediana \\
\hline Código da CID-10 & Descrição & & & \\
\hline F20-F29 & Esquizofrenia, transtornos esquizotípicos e delirantes & 6,23 & 4,046 & 7,0 \\
\hline F30-F39 & Transtornos de humor (afetivos) & 4,31 & 3,766 & 4,00 \\
\hline F40-F48 & $\begin{array}{c}\text { Transtornos neuróticos, transtornos relacionados } \\
\text { com o estresse e transtornos somatoformes }\end{array}$ & 5,05 & 3,768 & 4,00 \\
\hline F50-F59 & $\begin{array}{l}\text { Síndromes comportamentais associadas 6,37 } \\
\text { com distúrbios fisiológicos e a fatores físicos }\end{array}$ & 3,862 & 7,00 & \\
\hline F70-F79 & Retardo mental & 6,28 & 3,756 & 6,00 \\
\hline F80-F89 & Transtornos do desenvolvimento psicológico & 7,09 & 3,888 & 8,00 \\
\hline F90-F98 & $\begin{array}{l}\text { Transtornos do comportamento e transtornos emocionais } \\
\text { que aparecem habitualmente na infância ou na adolescência }\end{array}$ & 4,97 & 3,780 & 4,00 \\
\hline F99 & Transtorno mental não especificado & 6,05 & 3,273 & 6,00 \\
\hline Total & & 5,39 & 3,870 & 5,00 \\
\hline
\end{tabular}

CID-10: Classificação Estatística Internacional de Doenças e Problemas Relacionados à Saúde, 10a Revisão (CID-10) 17.

Fonte: Banco de dados Sistema de Informações Ambulatoriais/Autorizações de Pagamento de Serviços de Alta Complexidade, Departamento de Informática do SUS, Ministério da Saúde, 2003.

blema ser registrado em muitas situações como um diagnóstico secundário, associado a outros transtornos. Já para a esquizofrenia e transtornos esquizotípicos o percentual de $3 \%$ está dentro do esperado para estes serviços, específicos para pessoas com transtornos mentais graves.

\section{Características do atendimento}

Os CAPSI registraram atendimento de diferentes faixas etárias, e de um conjunto amplo de transtornos mentais, exceto aqueles decorrentes do uso de substâncias psicoativas. Mesmo estando previsto nas normas do Ministério da Saúde, nenhum atendimento foi registrado para esta condição. A compreensão deste achado deve ser aprofundada, uma vez que diversos estudos demonstram uma alta prevalência destes problemas. Segundo a OMS 3, cerca de $10 \%$ da população dos centros urbanos de todo o mundo, independente de idade, sexo ou condição social, consomem abusivamente substâncias psicoativas.

Contudo, no estudo desenvolvido em ambulatório de saúde mental, em Salvador 19, também não foram observados atendimentos pelo uso de substâncias psicoativas. Os motivos da ausência dessa demanda pode ser por dificuldade de aces- so, aliada ao entendimento, por parte dos profissionais, da necessidade de capacitação técnica específica para a detecção desses problemas, provocando a conseqüente exclusão da clientela dos serviços. Outra possibilidade é o encaminhamento dessas crianças e jovens para serviços específicos como os CAPS ad - serviços dirigidos para o atendimento de pessoas com problemas decorrentes do uso de álcool e outras e outras substâncias psicoativas. Por outro lado, mesmo sendo muito recente a incorporação deste tema no campo da saúde pública, vale ressaltar que um dos CAPSI excluídos do estudo apresentou atendimento para esta clientela, apontado eventuais limites deste estudo na captação do fenômeno.

Supunha-se que a freqüência dos transtornos severos como transtornos de humor, esquizofrenia e autismo excedessem o percentual encontrado de $19,4 \%$, visto que a prioridade ao atendimento de transtornos severos e persistentes constitui o objetivo primeiro dos serviços. Tal achado pode refletir o processo de consolidação desta nova rede, considerando-se a história anterior de encaminhamentos de crianças e jovens com transtornos mentais para hospitais psiquiátricos ou serviços de outras redes de atenção como educação, justiça e assistência social 10,11. 
Esperava-se encontrar um percentual maior de pessoas vinculadas ao modo intensivo de tratamento, pois tal modalidade de atenção estaria em princípio "reservada" para aqueles com transtornos mais graves, necessitando de acompanhamento intensivo, durante os cinco dias da semana. Por outro lado, a vinculação à determinada modalidade de atendimento não depende apenas do diagnóstico, incluindo recursos pessoais, familiares e sociais disponíveis, capazes de favorecer a permanência do indivíduo na comunidade.

As modalidades semi-intensivo e não intensivo absorveram o maior número de pessoas, demonstrando que a maioria dos serviços ainda funciona segundo a lógica ambulatorial tradicional em relação à vinculação da clientela, a qual não acontece de forma continuada e sistemática. A clientela vinculada à modalidade intensiva não ultrapassou $10 \%$ dos atendimentos, na maioria dos serviços. Vários fatores podem colaborar para esta forma de funcionamento, desde dificuldades materiais do usuário para obter recursos financeiros de transporte passando pela disponibilidade de acompanhante adulto em se tratando de crianças, até as dificuldades da própria equipe para estruturar um novo modelo de assistência.

\section{Aspectos administrativos}

Predominou a gestão municipal e o cumprimento da legislação quanto ao número de profissionais de nível superior e à área de abrangência, apesar da ausência de enfermeiro em um serviço, e um número menor de profissionais de nível médio em cinco unidades registradas no CNES. Por outro lado não foi possível verificar a periodicidade da atualização destas informações na página eletrônica do CNES.

Observou-se também que em 2003 os CAPSI não atenderam em sua capacidade plena. Isso se deve, possivelmente, ao fato de 2003 ter sido o primeiro ano de implantação dos serviços, associado à proposta de uma nova lógica de registro dos atendimentos (sistema SIA/APAC).

Ainda que a expansão, a avaliação e o acompanhamento das unidades sejam necessários para consolidação de uma rede substitutiva, os atuais CAPSI estão preenchendo uma lacuna histórica na atenção em saúde mental infanto-juvenil no SUS. Conseguiram em um curto espaço de tempo, atender uma demanda que contempla o conjunto de transtornos psiquiátricos, esboçando uma forma de atenção de base comunitária, contrapondo-se assim ao modelo hospitalocêntrico.

\section{Limitações do estudo}

Por mais que os resultados indiquem elementos importantes para o planejamento de ações e programas, cabe referir que este estudo utilizou dados secundários do sistema SIA/APAC, cujo objetivo do registro de informações é pautado por necessidades administrativas, para o pagamento de procedimentos referentes aos atendimentos realizados.

Tomaram-se como objeto de estudo apenas os diagnósticos conferidos por ocasião do início do atendimento, desconhecendo-se eventuais diagnósticos referentes às co-morbidades, ou ainda alterações diagnósticas durante o período de acompanhamento nos serviços.

Além disso, a exclusão de 25 unidades do universo de CAPSI elegíveis pode ter influenciado os achados, devido aos aspectos não contemplados na amostra estudada; porém a distribuição de sexo e idade entre os dois grupos de unidades foi semelhante. Mesmo assim optou-se pela utilização deste banco de dados, por ser a única fonte com dados nacionais disponível.

\section{Considerações finais}

Um sistema de informações sobre atendimentos em saúde mental infanto-juvenil deve incluir informações que contemplem dados sobre aspectos sociais, história de vida, co-morbidades, e interações com outras redes de atenção para enriquecer as análises e favorecer a decisão de gestores, planejadores, profissionais e organizações sociais.

A ausência de registros em unidades de um banco de dados vinculado ao repasse de recursos financeiros demonstra a necessidade de orientação dos profissionais quanto à importância da documentação. Os serviços que não realizaram registro no sistema SIA/APAC, no período estudado, não deixaram de realizar atendimentos à clientela infanto-juvenil, apesar de não terem recebido recursos do Ministério da Saúde pela atenção oferecida.

Recomenda-se o aprimoramento do sistema de registro de informações sobre estes serviços, e investimentos na formação dos recursos humanos especialmente para atender problemas decorrentes do uso de álcool e outras drogas na população infanto-juvenil, assim como a realização de estudos sobre a utilização e a qualidade dos serviços em saúde mental infantil, a fim de subsidiar o planejamento de expansão desta rede e as articulações intersetoriais. 


\section{Resumo}

Este estudo permitiu conhecer características diagnósticas, demográficas e procedimentos terapêuticos relativos à clientela dos Centros de Atenção Psicossocial Infanto-Juvenil (CAPSI), no Brasil, em 2003. Estudo descritivo, de corte transversal, com dados secundários. A análise considerou três conjuntos de variáveis: (a) relacionadas à natureza dos serviços; (b) clientela atendida; e (c) modalidades de cuidado oferecido. Participaram do estudo sete CAPSI que registraram atendimento nos 12 meses do ano de 2003. Esses serviços atenderam 1.456 pessoas, a maioria (62,8\%) do sexo masculino e tinham em média 11,1 anos. A modalidade de atendimento predominante foi o não intensivo (49,3\%). Os diagnósticos mais freqüentes foram dos transtornos do comportamento e transtornos emocionais (44,5\%). Não houve registro de atendimento decorrente do uso de substâncias psicoativas. O percentual de transtornos graves foi de 19,4\%. Os CAPSI apresentaram funcionamento semelhante aos serviços ambulatoriais, porém demonstraram atender uma demanda variada, cumprindo, em um curto espaço de tempo, as normas relacionadas ao registro das informações, conforme as modalidades de atendimento preconizadas pelo Ministério da Saúde, ocupando um vazio histórico na atenção pública em saúde mental infanto-juvenil.

Serviços de Saúde Mental; Criança; Adolescente; Saúde Mental

\section{Colaboradores}

M. C. C. L. Hoffmann participou do desenho do método do estudo, coleta dos dados, plano de análise, escrita e edição final do artigo. D. N. Santos colaborou no desenho de estudo, plano de análise, escrita e edição final do artigo. E. L. A. Mota contribuiu no desenvolvimento do plano de análise escrita e edição final do artigo.

\section{Agradecimentos}

Ao Ministério da Saúde pelo financiamento do projeto que conduziu a primeira autora à conclusão do Mestrado Profissional em Saúde Coletiva, realizado em parceria com o Instituto de Saúde Coletiva, Universidade Federal da Bahia.

\section{Referências}

1. Bordin IAS, Paula CS. Estudos populacionais sobre saúde mental de crianças e adolescentes brasileiros. In: Mello MF, Mello AAF, Kohn R, organizadores. Epidemiologia da saúde mental no Brasil. Porto Alegre: Editora Artmed; 2007. p. 101-17.

2. Fleitlich BW; Goodman R. Implantação e implementação de serviços de saúde mental comunitários para crianças e adolescentes. Rev Bras Psiquiatr 2002; 24:2.

3. Organização Mundial da Saúde. Relatório sobre saúde no mundo 2001. Saúde mental: nova concepção, nova esperança - 2001. Genebra: Organização Mundial da Saúde; 2001.

4. Almeida Filho N. Estudo de prevalência de desordens mentais na infância em uma zona urbana de Salvador. J Bras Psiquiatr 1982; 31:225-36.

5. Fleitlich-Bilyk B, Goodman R. Prevalence of child and adolescent psychiatric disorders in southeast Brazil. J Am Acad Child Adolesc Psychiatry 2004; 43:727-34.
6. Goodman R, Neves-dos-Santos D, RobattoNunes AP, Pereira-de-Miranda D, Fleitlich-Bilyk B, Almeida Filho N. The Ilha de Maré study: a survey of child mental health problems in a predominantly African-Brazilian rural community. Soc Psychiatry Psychiatr Epidemiol 2005; 40:11-7.

7. Soares KVS, Almeida Filho N, Botega NJ, Coutinho ESF, Mari JJ. Sintomas depressivos em adolescentes: análise dos dados do estudo multicêntrico de morbidade psiquiátrica em áreas metropolitanas Rev ABP-APAL 1994; 16:11-7.

8. Burke KC, Burke JD, Rae DS, Regier DA. Comparing age at onset of major depression and other psychiatric disorders by birth cohorts in five US community populations. Arch Gen Psychiatry 1991; 48:789-95.

9. Pelisoli CL, Moreira AK. Caracterização epidemiológica dos usuários do Centro de Atenção Psicossocial Casa Aberta. Rev Psiquiatr Rio Gd Sul 2005; 27:270-7. 
10. Schechtman A. Psiquiatria e infância: uma abordagem histórica. In: Russo J, Silva Filho JF, organizadores. Duzentos anos de psiquiatria. Rio de Janeiro: Editora Relume-Dumará/Editora da Universidade Federal do Rio de Janeiro; 1992. p. 85-93.

11. Rizzini I, Rizzini I. A institucionalização de crianças no Brasil: percurso histórico e desafios do presente. Rio de Janeiro: Editora PUC-Rio/São Paulo: Edições Loyola; 2004.

12. Tenório F. A reforma psiquiátrica brasileira, da década de 1980 aos dias atuais: história e conceitos. Hist Ciênc Saúde-Manguinhos 2002; 9:25-59.

13. Ministério da Saúde. Legislação em saúde mental 1990-2004. 5a Ed. Brasília: Ministério da Saúde; 2004.

14. Delgado PG. Economia solidária e saúde mental. In: Coordenação da Área Técnica de Saúde Mental, organizador. Saúde mental e economia solidária: inclusão social pelo trabalho. Brasília: Ministério da Saúde; 2005. p. 15-9.

15. Ministério da Saúde. Saúde mental no SUS: os Centros de Atenção Psicossocial. Brasília: Ministério da Saúde; 2004.
16. Delgado PG, Schechtman A, Amstalden AF, Bonavigo E, Cordeiro F, Porto K, et al. Reforma Psiquiátrica e política de Saúde Mental no Brasil - Conferência Regional de Reforma dos Serviços de Saúde Mental: 15 anos depois de Caracas. In: Mello MF, Mello AAF, Kohn R, organizadores. Epidemiologia da saúde mental no Brasil. Porto Alegre: Editora Artmed; 2007. p. 39-83.

17. Organização Mundial da Saúde. Classificação estatística internacional de doenças e problemas relacionados à saúde, 10a revisão. v. 1. São Paulo: Centro Colaborador da OMS para a Classificação de Doenças em Português; 1995.

18. Fundo de População das Nações Unidas. Direitos da população jovem: um marco para o desenvolvimento. Brasília: Fundo de População das Nações Unidas; 2003.

19. Santos DN, Carvalho MM, Pinho MA, Nunes APR. Atendimento em psiquiatria da infância e adolescência em serviços públicos de Salvador. Revista Baiana de Saúde Pública 2005; 29:35-42.

Recebido em 07/Nov/2006

Versão final reapresentada em 18/Abr/2007

Aprovado em 22/Mai/2007 